\title{
Micro-/Nano-Structures Fabricated by Laser Technologies for Optoelectronic Devices
}

Jian Yi, Hao Zhou, Wei-Hua Wei, Xing-Chen Han, Dong-Dong Han* and Bing-Rong Gao*

State Key Laboratory of Integrated Optoelectronics, College of Electronic Science and Engineering, Jilin University, Changchun, China

Due to unique optical and electrical properties, micro-/nano-structures have become an essential part of optoelectronic devices. Here, we summarize the recent developments in micro-/nano-structures fabricated by laser technologies for optoelectronic devices. The fabrication of micro-/nano-structures by various laser technologies is reviewed. Micro-/nano-structures in optoelectronic devices for performance improvement are reviewed. In addition, typical optoelectronic devices with micro-nano structures are also summarized. Finally, the challenges and prospects are discussed.

Keywords: micro-/nano-structures, laser technologies, photodetector, photovoltaic cell, light-emitting diode

\section{INTRODUCTION}

There are many animals and plants using unique micro-/nano-structures to improve their environmental adaptability (Han et al., 2016; Han et al., 2020a; Cao et al., 2020). For example, micro-/nano-structures on a lotus leaf and taro surface exhibit superhydrophobic properties (Zhang et al., 2012b; Wang et al., 2021b; Lv et al., 2021). Grating-like structures on butterfly wings trap light, leading to the colorful butterfly wing (Wang et al., 2012; Jiang et al., 2016; Zou et al., 2020). Learning from nature, micro-/nano-structures have been adopted in the various functional devices for broad applications (Han et al., 2019; Zhang et al., 2019; Zhang et al., 2021). Therefore, many researchers have focused on the fabrication and application of micro-nano structures (Zhang et al., 2012a; Han et al., 2015; Liu et al., 2021). Mainly, due to unique optical and electrical properties, micro-/nano-structures have become an essential part of optoelectronic devices.

Laser fabrication technologies show high efficiency, high precision, and low thermal effect (Li et al., 2020a; Fu et al., 2020; Ma et al., 2020; You et al., 2020; Fu et al., 2021). Laser technologies can be used to fabricate micro-/nano-structures by the interaction between laser and materials (Liu et al., 2019; Han et al., 2020b; Liu et al., 2020; Wang et al., 2021a). Especially, ultrafast lasers can fabricate broadband, transparent anti-reflection surfaces, which promote the performance of optoelectronic devices by enhancing the light absorption or introducing surface plasmon-polariton (Zhang et al., 2010; Liapis et al., 2017; Jia et al., 2020).

In this review, we summarize recent progress on micro-/nano-structures fabricated by laser technologies. Typical light trapping mechanism and surface plasmon-polariton of the micro-nano structure are discussed. Then, we outlined the typical applications, including photodetectors, photovoltaic cells, organic light-emitting devices, etc. Finally, the challenges and prospects are discussed. 


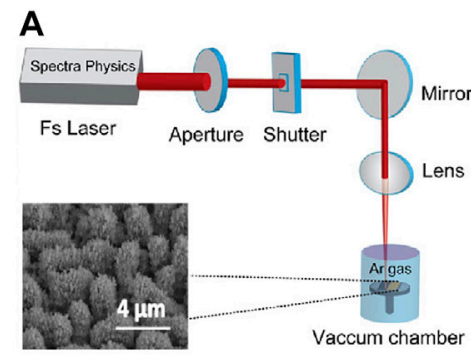

D

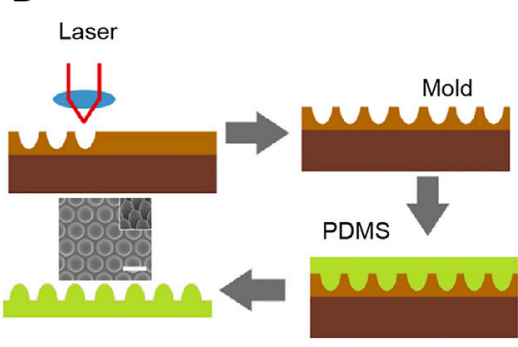

G

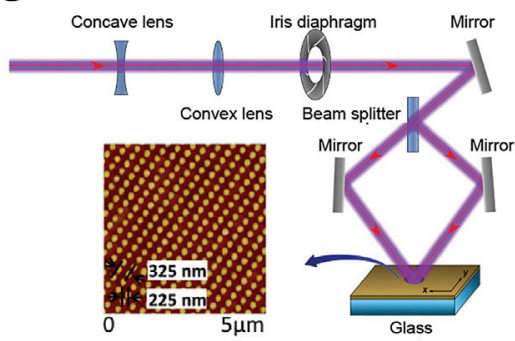

B

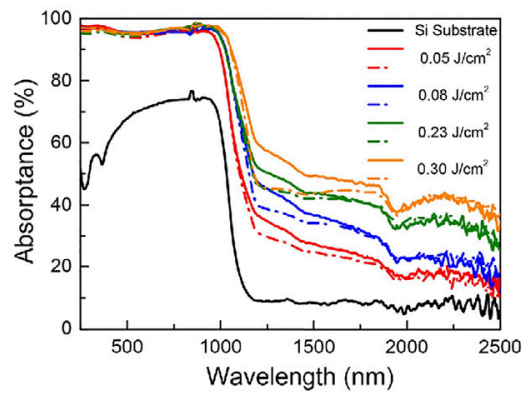

E

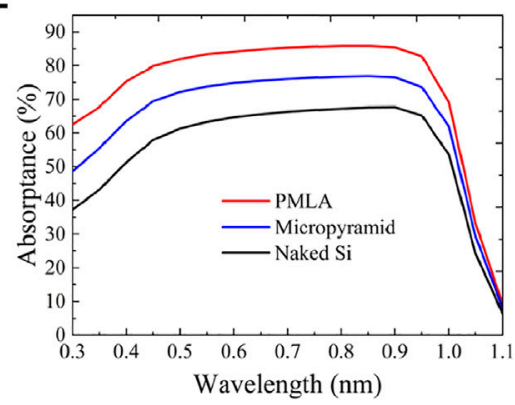

H

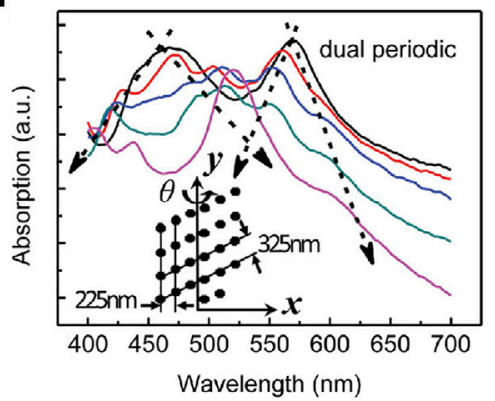

C

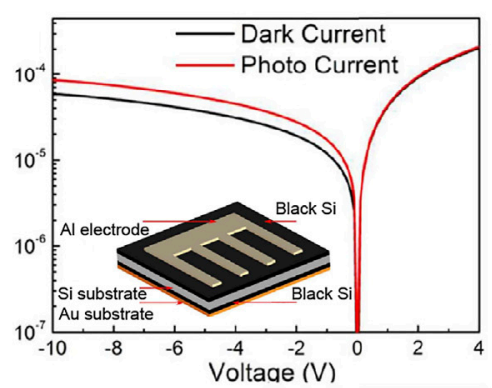

F

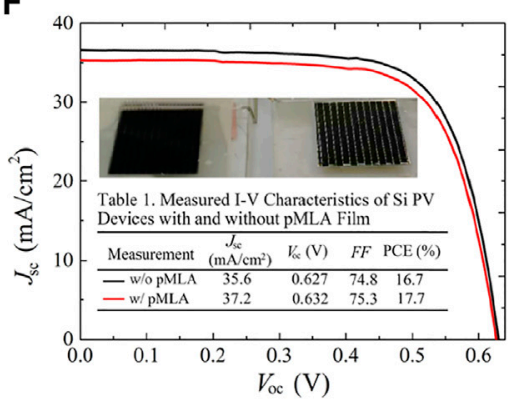

I

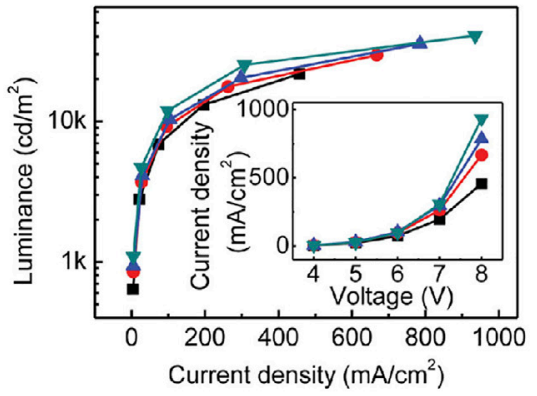

FIGURE 1 | Micro-/nano-structures fabricated by laser technologies for optoelectronic devices. (A) Femtosecond laser manufacturing system. Reproduced from (Yu et al., 2017) with permission of IEEE. (B) The corresponding absorptivity of nitrogen-doped silicon substrate. (C) Infrared photodetection performance. Reproduced from (Li et al., 2018) with permission of IEEE. (D) The preparation process of PDMS convex PMLA film. (E) Simulated absorption spectra of films. (F) Solar cell performance. Reproduced from (Fang et al., 2018) with permission of American Chemical Society. (G) Schematic diagram of laser two-beam interferes processing. Reproduced from (Bi et al., 2013; Jiang et al., 2014) with permission of WILEY-VCH. (H) Absorption spectra for WOLEDs. (I) Performance of WOLED. Reproduced from (Bi et al., 2013) with permission of WILEY-VCH.

\section{MECHANISM}

Introducing micro-/nano-structures inside or outside the devices can improve optoelectronic devices' performance (Ma and Cui, 2020; Na and Chew, 2020; Chen et al., 2021). Inspired by the moth-eye structure, the reflectivity is reduced by introducing micro-/nano-structures. Mainly, the light will be internally reflected many times inside the structure to form a "light trap" (Zhang et al., 2020a; Otte and Denz, 2020; Yang et al., 2021). As a result, the existence of micro-/nanostructures can improve the light absorption capacity of the optoelectronic device. Moreover, the efficiency of optoelectronic devices can be enhanced by surface plasmonpolariton (Eaton et al., 2016; Li et al., 2020b; Zhang et al., 2020b).

\section{OPTOELECTRONIC DEVICES}

\section{Photodetector}

Silicon material plays an important role in silicon-based optoelectronic integrated devices preparation. Take photodetectors as an example, the bandgap of silicon material is around $1.1-1.3 \mathrm{eV}$, limiting silicon material for infrared radiation (IR) photodetection. Therefore, many efforts, such as ion implantation or structural defects, have been developed to extend the absorption band of silicon. As a pioneer, Zhao's group (Li et al., 2017; Yu et al., 2017; Li et al., 2018) fabricated supersaturated silicon material with nitrogen, sulfur, and Au by femtosecond laser ablation (Figure 1A). After the femtosecond laser ablation in nitrogen $\left(\mathrm{N}_{2}\right)$ atmosphere, the surface silicon material evolved into a bead-like micro-/nano-structures with a height of $3 \sim 4 \mu \mathrm{m}$ and a distance of 
3 4 $\mu \mathrm{m}$ (Li et al., 2018). Micro-/nano-structures are beneficial for a stronger light trapping effect. Compared with the initial silicon material, laser-treated $\mathrm{N}$-doped silicon material has a broader absorption $(0.25-2.5 \mu \mathrm{m})$ and higher absorptivity (Figure 1B). The inset of Figure 1C is the device structure of the laser-treated silicon-based IR photodetector. The photo responsivity is $5.3 \mathrm{~mA} / \mathrm{W}(\mathrm{V}=10 \mathrm{~V})$.

\section{Photovoltaic Cell}

Photovoltaic cells convert sunlight to electric energy. Usually, light utilization efficiency is very low due to the reflection loss. To solve this problem, various anti-reflection structures have been designed. For example, Fang et al. proposed a 100\% relative packing density film for enhancing photovoltaic cells performance (Fang et al., 2018). As shown in Figure 1D, direct-write ultraviolet (UV) laser photolithography system was employed to fabricate a paraboloidal concave photoresist pattern (master mold). Then polydimethylsiloxane (PDMS) was spin-coated onto the master mold. After thermally cured, structured PDMS was separated from the master mold for further use. Figure $1 \mathrm{E}$ is the simulated absorption spectra of films. Si substrate with paraboloidal microlens array (PMLA) film shows the highest absorption due to the suppressing reflection. It is worth noting that PMLA antireflective (AR) film indicates superhydrophobicity and self-cleaning ability. Finally, the shortcircuit current density increases from 35.6 to $37.2 \mathrm{~mA} / \mathrm{cm}^{2}$ after integrating the PMLA AR film (Figure 1F). Instead of integrating the AR film on the photovoltaic cells surface, structured photoelectrodes or active layers have been fabricated by femtosecond laser ablation, interference, or laser-induced periodic surface structures for photocurrent enhancement (Zhang et al., 2015; Cui et al., 2016; Soldera et al., 2016).

\section{Light-Emitting Diode}

Bi et al. (2013) demonstrated white organic light-emitting diodes (WOLEDs) with broadband excitation by introducing twodimensional gratings. As shown in Figure 1G, the grating structures were prepared by two-beam laser interference (Guo et al., 2012; Jiang et al., 2014; Yan et al., 2015). Introducing dualperiod corrugations into the WOLED metal electrodes achieves broadband absorption (Figure $\mathbf{1 H}$ ). In addition, broadband SPP modes lead to broadband light extraction. Significantly, broadband light extraction deeply affects the WOLEDs

\section{REFERENCES}

Bi, Y.-G., Feng, J., Li, Y.-F., Zhang, X.-L., Liu, Y.-F., Jin, Y., et al. (2013). Broadband Light Extraction from white Organic Light-Emitting Devices by Employing Corrugated Metallic Electrodes with Dual Periodicity. Adv. Mater. 25 (48), 6969-6974. doi:10.1002/adma.201302367

Cao, J.-J., Hou, Z.-S., Tian, Z.-N., Hua, J.-G., Zhang, Y.-L., and Chen, Q.-D. (2020). Bioinspired Zoom Compound Eyes Enable Variable-Focus Imaging. ACS Appl. Mater. Inter. 12 (9), 10107-10117. doi:10.1021/ acsami.9b21008

Chen, J., Zhou, Y., Fu, Y., Pan, J., Mohammed, O. F., and Bakr, O. M. (2021). Oriented Halide Perovskite Nanostructures and Thin Films for performance (Figure 1I). Compared with traditional planar devices, the current efficiency is increased by $37 \%$, and the external quantum efficiency is increased by $48 \%$. Recently, combining laser interference lithography and reactive ion etching, Ju et al. proposed flexible OLEDs with light extraction structure for optical efficiency improvement (Lee et al., 2019; Kim et al., 2020).

\section{CONCLUSION AND OUTLOOK}

This minireview summarizes recent progress on micro-/nanostructures fabricated by laser technologies for optoelectronic devices. The existence of micro-/nano-structures can improve the light absorption capacity and the efficiency of optoelectronic devices. Typical optoelectronic devices have been successfully designed and demonstrated the critical role of micro-/nanostructures. Significantly, new photoelectric applications, such as photoelectric dichroism, have been proposed and fabricated by laser technology based on various materials (Drevinskas et al., 2015; Jiang et al., 2020; Kuroiwa and Tatsuma, 2020; Zou et al., 2021a; Zou et al., 2021b; Xuan et al., 2021). Although successful works have demonstrated the distinguish characters, the efficiency of laser processing materials needs to improve, which benefits device preparation efficiency. With the rapid development of nanofabrication technology, advanced fundamental theories, new structural design, micro-/nanostructures will improve devices performances.

\section{AUTHOR CONTRIBUTIONS}

All authors listed have made a substantial, direct, and intellectual contribution to the work and approved it for publication.

\section{FUNDING}

This research was funded by the Strategic Priority Research Program of CAS (Grant No. XDC07030303), the National Natural Science Foundation of China (61905087), and Fundamental Research Funds for the Central Universities (2020-JCXK-18).

Optoelectronics. Chem. Rev. 121 (20), 12112-12180. doi:10.1021/ acs.chemrev.1c00181

Cui, J., Rodríguez-Rodríguez, Á., Hernández, M., García-Gutiérrez, M.-C., Nogales, A., Castillejo, M., et al. (2016). Laser-induced Periodic Surface Structures on P3HT and on its Photovoltaic Blend with PC71BM. ACS Appl. Mater. Inter. 8 (46), 31894-31901. doi:10.1021/acsami.6b09053

Drevinskas, R., Beresna, M., Gecevičius, M., Khenkin, M., Kazanskii, A. G., Matulaitiené, I., et al. (2015). Giant Birefringence and Dichroism Induced by Ultrafast Laser Pulses in Hydrogenated Amorphous Silicon. Appl. Phys. Lett. 106 (17), 171106. doi:10.1063/1.4919538

Eaton, S. W., Fu, A., Wong, A. B., Ning, C.-Z., and Yang, P. (2016). Semiconductor Nanowire Lasers. Nat. Rev. Mater. 1 (6), 16028. doi:10.1038/ natrevmats.2016.28 
Fang, C., Zheng, J., Zhang, Y., Li, Y., Liu, S., Wang, W., et al. (2018). Antireflective Paraboloidal Microlens Film for Boosting Power Conversion Efficiency of Solar Cells. ACS Appl. Mater. Inter. 10 (26), 21950-21956. doi:10.1021/ acsami.7b19743

Fu, X.-Y., Cai, Q., Ma, J.-N., Zhu, L., Han, D.-D., and Zhang, Y.-L. (2021). Freestanding and Flexible Graphene Supercapacitors of High Areal Capacitance Fabricated by Laser Holography Reduction of Graphene Oxide. Appl. Phys. Lett. 118 (7), 071601. doi:10.1063/5.0038508

Fu, X.-Y., Chen, Z.-D., Han, D.-D., Zhang, Y.-L., Xia, H., and Sun, H.-B. (2020). Laser Fabrication of Graphene-Based Supercapacitors. Photon. Res. 8 (4), 577-588. doi:10.1364/prj.382401

Guo, L., Jiang, H.-B., Shao, R.-Q., Zhang, Y.-L., Xie, S.-Y., Wang, J.-N., et al. (2012). Two-beam-laser Interference Mediated Reduction, Patterning and Nanostructuring of Graphene Oxide for the Production of a Flexible Humidity Sensing Device. Carbon 50 (4), 1667-1673. doi:10.1016/ j.carbon.2011.12.011

Han, B., Zhang, Y. L., Zhu, L., Li, Y., Ma, Z. C., Liu, Y. Q., et al. (2019). PlasmonicAssisted Graphene Oxide Artificial Muscles. Adv. Mater. 31 (5), 1806386. doi:10.1002/adma.201806386

Han, D.-D., Cai, Q., Chen, Z.-D., Li, J.-C., Mao, J.-W., Lv, P., et al. (2020a). Bioinspired Surfaces with Switchable Wettability. Front. Chem. 8, 692. doi:10.3389/fchem.2020.00692

Han, D.-D., Chen, Z.-D., Li, J.-C., Mao, J.-W., Jiao, Z.-Z., Wang, W., et al. (2020b). Airflow Enhanced Solar Evaporation Based on Janus Graphene Membranes with Stable Interfacial Floatability. ACS Appl. Mater. Inter. 12, 25435-25443. doi:10.1021/acsami.0c05401

Han, D.-D., Zhang, Y.-L., Liu, Y., Liu, Y.-Q., Jiang, H.-B., Han, B., et al. (2015). Bioinspired Graphene Actuators Prepared by Unilateral UV Irradiation of Graphene Oxide Papers. Adv. Funct. Mater. 25 (28), 4548-4557. doi:10.1002/ adfm.201501511

Han, D.-D., Zhang, Y.-L., Ma, J.-N., Liu, Y.-Q., Han, B., and Sun, H.-B. (2016). Light-mediated Manufacture and Manipulation of Actuators. Adv. Mater. 28 (38), 8328-8343. doi:10.1002/adma.201602211

Jia, L., Zheng, W., and Huang, F. (2020). Vacuum-ultraviolet Photodetectors. PhotoniX 1 (1), 22. doi:10.1186/s43074-020-00022-w

Jiang, H.-B., Zhang, Y.-L., Han, D.-D., Xia, H., Feng, J., Chen, Q.-D., et al. (2014). Bioinspired Fabrication of Superhydrophobic Graphene Films by Two-Beam Laser Interference. Adv. Funct. Mater. 24 (29), 4595-4602. doi:10.1002/ adfm.201400296

Jiang, H.-B., Zhang, Y.-L., Liu, Y., Fu, X.-Y., Li, Y.-F., Liu, Y.-Q., et al. (2016). Bioinspired Few-Layer Graphene Prepared by Chemical Vapor Deposition on Femtosecond Laser-Structured Cu Foil. Laser Photon. Rev. 10 (3), 441-450. doi:10.1002/lpor.201500256

Jiang, H., Zhao, B., Liu, Y., Li, S., Liu, J., Song, Y., et al. (2020). Review of Photoreduction and Synchronous Patterning of Graphene Oxide toward Advanced Applications. J. Mater. Sci. 55 (2), 480-497. doi:10.1007/s10853-019-03981-z

Kim, J. G., Lee, J. S., Hwang, H., Kim, E., Choi, Y., Kwak, J. H., et al. (2020). Modeling of Flexible Light Extraction Structure: Improved Flexibility and Optical Efficiency for Organic Light-Emitting Diodes. Org. Electronics 85, 105760. doi:10.1016/j.orgel.2020.105760

Kuroiwa, Y., and Tatsuma, T. (2020). Laser Printing of Translucent Plasmonic Full-Color Images with Transmission-Scattering Dichroism of Silver Nanoparticles. ACS Appl. Nano Mater. 3 (3), 2472-2479. doi:10.1021/ acsanm. $9 \mathrm{~b} 02560$

Lee, J. S., Shim, Y. S., Park, C. H., Hwang, H., Park, C. H., Joo, C. W., et al. (2019). Enhanced Light Extraction from Organic Light-Emitting Diodes Using a QuasiPeriodic Nano-Structure. Nanotechnology 30 (8), 085302. doi:10.1088/13616528/aaf541

Li, C.-H., Wang, X.-P., Zhao, J.-H., Zhang, D.-Z., Yu, X.-Y., Li, X.-B., et al. (2018). Black Silicon IR Photodiode Supersaturated with Nitrogen by Femtosecond Laser Irradiation. IEEE Sensors J. 18 (9), 3595-3601. doi:10.1109/ jsen.2018.2812730

Li, C.-H., Zhao, J.-H., Yu, X.-Y., Chen, Q.-D., Feng, J., Han, P.-D., et al. (2017). Sulfur-doped Silicon Photodiode by Ion Implantation and Femtosecond Laser Annealing. IEEE Sensors J. 17 (8), 2367-2371. doi:10.1109/jsen.2017.2666178

Li, Z.-Z., Wang, L., Fan, H., Yu, Y.-H., Chen, Q.-D., Juodkazis, S., et al. (2020a). O-FIB: Far-Field-Induced Near-Field Breakdown for Direct Nanowriting in an
Atmospheric Environment. Light Sci. Appl. 9 (1), 41. doi:10.1038/s41377-0200275-2

Li, Z., Xu, B., Liang, D., and Pan, A. (2020b). Polarization-dependent Optical Properties and Optoelectronic Devices of 2D Materials. Research 2020, 1-35. doi:10.34133/2020/5464258

Liapis, A. C., Rahman, A., and Black, C. T. (2017). Self-assembled Nanotextures Impart Broadband Transparency to Glass Windows and Solar Cell Encapsulants. Appl. Phys. Lett. 111 (18), 183901. doi:10.1063/1.5000965

Liu, Y.-Q., Chen, Z.-D., Mao, J.-W., Han, D.-D., and Sun, X. (2019). Laser Fabrication of Graphene-Based Electronic Skin. Front. Chem. 7, 461. doi: $10.3389 /$ fchem. 2019.00461

Liu, Y.-Q., Mao, J.-W., Chen, Z.-D., Han, D.-D., Jiao, Z.-Z., Ma, J.-N., et al. (2020). Three-dimensional Micropatterning of Graphene by Femtosecond Laser Direct Writing Technology. Opt. Lett. 45 (1), 113-116. doi:10.1364/ol.45.000113

Liu, Y. Q., Chen, Z. D., Han, D. D., Mao, J. W., Ma, J. N., Zhang, Y. L., et al. (2021). Bioinspired Soft Robots Based on the Moisture-Responsive Graphene Oxide. Adv. Sci. 8, 2002464. doi:10.1002/advs.202002464

Lv, P., Zhang, Y. L., Han, D. D., and Sun, H. B. (2021). Directional Droplet Transport on Functional Surfaces with Superwettabilities. Adv. Mater. Inter. 8 (12), 2100043. doi:10.1002/admi.202100043

Ma, Q., and Cui, T. J. (2020). Information Metamaterials: Bridging the Physical World and Digital World. PhotoniX 1 (1), 1. doi:10.1186/s43074-020-00006-w

Ma, Z.-C., Zhang, Y.-L., Han, B., Hu, X.-Y., Li, C.-H., Chen, Q.-D., et al. (2020). Femtosecond Laser Programmed Artificial Musculoskeletal Systems. Nat. Commun. 11 (1), 4536. doi:10.1038/s41467-020-18117-0

Na, D.-Y., and Chew, W. C. (2020). Classical and Quantum Electromagnetic Interferences: What Is the Difference? Pier 168, 1-13. doi:10.2528/PIER20060301

Otte, E., and Denz, C. (2020). Optical Trapping Gets Structure: Structured Light for Advanced Optical Manipulation. Appl. Phys. Rev. 7 (4), 041308. doi:10.1063/ 5.0013276

Soldera, M., Taretto, K., Berger, J., and Lasagni, A. F. (2016). Potential of Photocurrent Improvement in $\mu \mathrm{c}-\mathrm{Si}: \mathrm{H}$ Solar Cells with TCO Substrates Structured by Direct Laser Interference Patterning. Adv. Eng. Mater. 18 (9), 1674-1682. doi:10.1002/adem.201600225

Wang, H., Xu, B.-B., Zhang, Y.-L., Kollipara, P. S., Liu, S., Lin, L., et al. (2021a). Light-driven Magnetic Encoding for Hybrid Magnetic Micromachines. Nano Lett. 21 (4), 1628-1635. doi:10.1021/acs.nanolett.0c04165

Wang, H., Zhang, Y.-L., Han, D.-D., Wang, W., and Sun, H.-B. (2021b). Laser Fabrication of Modular Superhydrophobic Chips for Reconfigurable Assembly and Self-Propelled Droplet Manipulation. PhotoniX 2 (1), 17. doi:10.1186/ s43074-021-00033-1

Wang, J.-N., Shao, R.-Q., Zhang, Y.-L., Guo, L., Jiang, H.-B., Lu, D.-X., et al. (2012). Biomimetic Graphene Surfaces with Superhydrophobicity and Iridescence. Chem. Asian J. 7 (2), 301-304. doi:10.1002/asia.201100882

Xuan, Z., Li, J., Liu, Q., Yi, F., Wang, S., and Lu, W. (2021). Artificial Structural Colors and Applications. The Innovation 2 (1), 100081. doi:10.1016/ j.xinn.2021.100081

Yan, Z.-X., Zhang, Y.-L., Wang, W., Fu, X.-Y., Jiang, H.-B., Liu, Y.-Q., et al. (2015). Superhydrophobic Sers Substrates Based on Silver-Coated Reduced Graphene Oxide Gratings Prepared by Two-Beam Laser Interference. ACS Appl. Mater. Inter. 7 (49), 27059-27065. doi:10.1021/acsami.5b09128

Yang, Y., Ren, Y.-X., Chen, M., Arita, Y., and Rosales-Guzmán, C. (2021). Optical Trapping with Structured Light: a Review. Adv. Photon. 3 (3), 034001. doi:10.1117/1.ap.3.3.034001

You, R., Liu, Y. Q., Hao, Y. L., Han, D. D., Zhang, Y. L., and You, Z. (2020). Laser Fabrication of Graphene-Based Flexible Electronics. Adv. Mater. 32, 1901981. doi:10.1002/adma.201901981

Yu, X.-Y., Zhao, J.-H., Li, C.-H., Chen, Q.-D., and Sun, H.-B. (2017). Goldhyperdoped Black Silicon with High IR Absorption by Femtosecond Laser Irradiation. IEEE Trans. Nanotechnology 16 (3), 502-506. doi:10.1109/ tnano.2017.2693691

Zhang, H. H., Wang, P. P., Zhang, S., Li, L., Li, P., Sha, W. E. I., et al. (2020a). Electromagnetic-circuital-thermal Multiphysics Simulation Method: a Review (Invited). Pier 169, 87-101. doi:10.2528/PIER20112801

Zhang, J., Hu, X., Chen, H., and Gao, F. (2020b). Designer Surface Plasmons Enable Terahertz Cherenkov Radiation (Invited). Pier 169, 25-32. doi:10.2528/ PIER20102708 
Si et al.

Laser Technologies for Optoelectronic Devices

Chang, X., Lu, H., Huang, X., and Jiang, H. (2015). One-step Femtosecond Laser Patterning of Light-Trapping Structure on Dye-Sensitized Solar Cell Photoelectrodes. J. Mater. Chem. C 3 (14), 3336-3341. doi:10.1039/c4tc02657h

Chang, Y.-L., Chen, Q.-D., Jim, Z., Kim, E., and Sun, H.-B. (2012a). Biomimetic Graphene Films and Their Properties. Nanoscale 4 (16), 4858-4869. doi:10.1039/c2nr30813d

Chang, Y.-L., Chen, Q.-D., Xia, H., and Sun, H.-B. (2010). Designable 3D Nanofabrication by Femtosecond Laser Direct Writing. Nano Today 5 (5), 435-448. doi:10.1016/j.nantod.2010.08.007

Chang, Y.-L., Li, J.-C., Zhou, H., Lu, Y.-Q., Han, D.-D., and Sun, H.-B. (2021). Electro-responsive Actuators Based on Grapheme. The Innovation 2 (4), 100168. doi:10.1016/j.xinn.2021.100168

Chang, Y.-L., Xia, H., Kim, E., and Sun, H.-B. (2012b). Recent Developments in Superhydrophobic Surfaces with Unique Structural and Functional Properties. Soft Matter 8 (44), 11217-11231. doi:10.1039/c2sm26517f

Chang, Y. L., Lu, Y. Q., Han, D. D., Ma, J. N., Wang, D., Li, X. B., et al. (2019). Quantum-Confined-Superfluidics-Enabled Moisture Actuation Based on Unilaterally Structured Grapheme Oxide Papers. Adv. Mater. 31 (32), 1901585. doi:10.1002/adma.201901585

Zoo, T., Zhao, B., Yin, W., Wang, F., Xe, H., Li, Y., et al. (2021a). Birefringent Response of Grapheme Oxide Film Structurized via Femtosecond Laser. Nan Res. 1, 1. doi:10.1007/s12274-021-3505-x

Zou, T., Zhao, B., Yin, W., Wang, Y., Wang, B., Zheng, X., et al. (2020). High-speed Femtosecond Laser Plasmonic Lithography and Reduction of Grapheme Oxide for Anisotropic Photoresponse. Light Sci. Apple. 9 (1), 69. doi:10.1038/s41377020-0311-2

Zou, X., Xu, Y., and Duan, W. (2021b). 2D Materials: Rising star for Future Applications. The Innovation 2 (2), 100115. doi:10.1016/ j.xinn.2021.100115

Conflict of Interest: The authors declare that the research was conducted in the absence of any commercial or financial relationships that could be construed as a potential conflict of interest.

Publisher's Note: All claims expressed in this article are solely those of the authors and do not necessarily represent those of their affiliated organizations, or those of the publisher, the editors and the reviewers. Any product that may be evaluated in this article, or claim that may be made by its manufacturer, is not guaranteed or endorsed by the publisher.

Copyright (c) 2021 Yin, Chou, Wei, Han, Han and Gao. This is an open-access article distributed under the terms of the Creative Commons Attribution License (CC BY). The use, distribution or reproduction in other forums is permitted, provided the original authors) and the copyright owners) are credited and that the original publication in this journal is cited, in accordance with accepted academic practice. No use, distribution or reproduction is permitted which does not comply with these terms.

Frontiers in Chemistry | www.frontiersin.org

5

December 2021 | Volume 9 | Article 823715 